\title{
Radiation from Relativistic Shocks in Turbulent Magnetic Fields
}

\author{
K.-I. Nishikawa ${ }^{\text {a }}$, J. Niemiec ${ }^{\mathrm{b}}$, M. Medvedev ${ }^{\mathrm{c}}$, B. Zhang ${ }^{\mathrm{d}}$, \\ P. Hardee ${ }^{\mathrm{e}}$, A. Nordlund $^{\mathrm{f}}$, J. Frederiksen ${ }^{\mathrm{f}}$, Y. Mizuno ${ }^{\mathrm{a}}$, \\ H. Sol ${ }^{g}$, M. Pohl ${ }^{h}$, D. H. Hartmann ${ }^{i}$, M. Oka ${ }^{j}$, and \\ G. J. Fishman ${ }^{k}$ \\ ${ }^{a}$ National Space Science and Technology Center, Huntsville, AL 35805, USA \\ ${ }^{\mathrm{b}}$ Institute of Nuclear Physics PAN, ul. Radzikowskiego 152, 31-342 Kraków, \\ Poland \\ ${ }^{\mathrm{c}}$ Department of Physics and Astronomy, University of Kansas, KS 66045, USA \\ ${ }^{\mathrm{d}}$ Department of Physics, University of Nevada, Las Vegas, NV 89154, USA \\ ${ }^{\mathrm{e}}$ Department of Physics and Astronomy, The University of Alabama, Tuscaloosa, \\ AL 35487, USA \\ ${ }^{\mathrm{f}}$ Niels Bohr Institute, University of Copenhagen, Juliane Maries Vej 30, 2100 \\ Copenhagen Ø, Denmark \\ ${ }^{g}$ LUTH, Observatore de Paris-Meudon, 5 place Jules Jansen, 92195 Meudon \\ Cedex, France \\ ${ }^{\mathrm{h}}$ Department of Physics and Astronomy, Iowa State University, Ames, IA 50011, \\ $U S A$ \\ i Department of Physics and Astronomy, Clemson University, Clemson, SC 29634, \\ $U S A$ \\ j 1 Space Sciences Laboratory, University of California, Berkeley, California 94720, \\ $U S A$ \\ ${ }^{\mathrm{k}} N A S A / M S F C$, Huntsville, AL 35805, USA
}

\begin{abstract}
Using our new 3-D relativistic particle-in-cell (PIC) code parallelized with MPI, we investigated long-term particle acceleration associated with a relativistic electronpositron jet propagating in an unmagnetized ambient electron-positron plasma. The simulations were performed using a much longer simulation system than our previous simulations in order to investigate the full nonlinear stage of the Weibel instability and its particle acceleration mechanism. Cold jet electrons are thermalized and ambient electrons are accelerated in the resulting shocks. Acceleration of ambient electrons leads to a maximum ambient electron density three times larger than the original value as predicted by hydrodynamic shock compression. In the
\end{abstract}


jet (reverse) shock behind the bow (forward) shock the strongest electromagnetic fields are generated. These fields may lead to time dependent afterglow emission. In order to calculate radiation from first principles that goes beyond the standard synchrotron model used in astrophysical objects we have used PIC simulations. Initially we calculated radiation from electrons propagating in a uniform parallel magnetic field to verify the technique. We then used the technique to calculate emission from electrons in a small simulation system. From these simulations we obtained spectra which are consistent with those generated from electrons propagating in turbulent magnetic fields with red noise. This turbulent magnetic field is similar to the magnetic field generated at an early nonlinear stage of the Weibel instability. A fully developed shock within a larger simulation system may generate a jitter/synchrotron spectrum.

Key words: acceleration of particles, galaxies, jets, gamma rays bursts, magnetic fields, plasmas, shock waves, radiation

\section{Introduction}

Particle-in-cell (PIC) simulations can shed light on the physical mechanism of particle acceleration that occurs in the complicated dynamics within relativistic shocks. Recent PIC simulations of relativistic electron-ion and electronpositron jets injected into an ambient plasma show that acceleration occurs within the downstream jet (Nishikawa et al., 2003, 2005; Hededal \& Nishikawa, 2005; Nishikawa et al., 2006; Ramirez-Ruiz. Nishikawa. \& Hededal, 2007; Chang. Spitkovsky, \& Arons, 2008; Spitkovsky, 2008a, b; ; Sironi \& Spitkovsky, 2009a). In general, these simulations have confirmed that the Weibel instability, which generates current filaments and associated magnetic fields mediates the relativistic collisionless shock (Medvedev, 1999), and accelerates electrons (Hededal \& Nishikawa, 2005; Nishikawa et al., 2006; Ramirez-Ruiz. Nishikawa, \& Hededal, 2007; Chang. Spitkovsky, \& Arons, 2008; Spitkovsky, 2008a, b; Sironi \& Spitkovsky, 2009a). Therefore, the investigation of radiation resulting from accelerated particles (mainly electrons and positrons) in turbulent magnetic fields is essential to understanding the radiation and observable spectral properties. In this article we present a numerical method to obtain spectra from particles self-consistently traced in our PIC simulations.

\section{Relativistic PIC Simulations}

\subsection{An Unmagnetized Pair Jet Injected into an Unmagnetized Pair Plasma}

We have performed a simulation using a system with $\left(L_{\mathrm{x}}, L_{\mathrm{y}}, L_{\mathrm{z}}\right)=(4005 \Delta$, $131 \Delta, 131 \Delta)(\Delta=1$ : grid size) and a total of $\sim 1$ billion particles (12 particles/cell/species for the ambient plasma) in the active grid zones (Nishikawa et al., 2009). In the simulation the electron skin depth, $\lambda_{\text {ce }}=c / \omega_{\text {pe }}=10.0 \Delta$, where $\omega_{\text {pe }}=\left(4 \pi e^{2} n_{\mathrm{e}} / m_{\mathrm{e}}\right)^{1 / 2}$ is the electron plasma frequency and the elec- 
tron Debye length $\lambda_{\mathrm{e}}$ is half of the grid size. Here the computational domain is six times longer than in our previous simulations (Nishikawa et al., 2006; Ramirez-Ruiz, Nishikawa, \& Hededal, 2007). The electron number density of the jet is $0.676 n_{\mathrm{e}}$, where $n_{\mathrm{e}}$ is the ambient electron density and $\gamma=15$. This parameter regime may be relevant to gamma-ray burst afterglows and AGN jets. The electron/positron thermal velocity of the jet is $v_{\mathrm{j}, \mathrm{th}}^{\mathrm{e}}=0.014 c$, where $c=1$ is the speed of light.

Figure 1 shows the averaged (in the $y-z$ plane) electron density and electromagnetic field energy along the jet at $3750 \omega_{\mathrm{pe}}^{-1}$. The resulting profiles of jet (red), ambient (blue), and total (black) electron density are shown in Fig. 1a. The ambient electrons are accelerated by the jet electrons and pile up towards

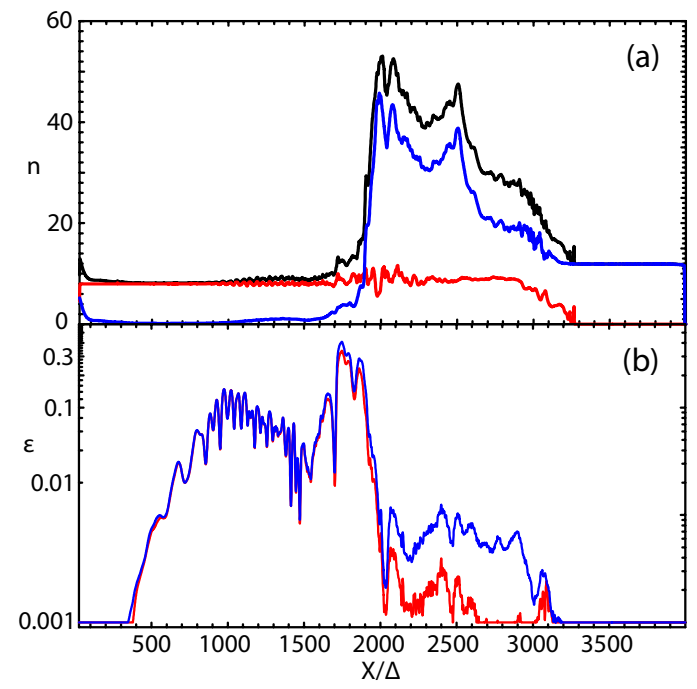

Fig. 1. The averaged values of electron density (a) and field energy (b) along the $x$ at $t=3750 \omega_{\mathrm{pe}}^{-1}$. Fig. 1a shows jet electrons (red), ambient electrons (blue), and the total electron density (black). Fig. 1b shows electric field energy (red) and magnetic field energy (blue) divided by the total kinetic energy.

the front part of jet. At earlier times the ambient plasma density increases linearly behind the jet front. At the later times the ambient plasma shows a rapid increase to a plateau behind the jet front, with additional increase to a higher plateau farther behind the jet front. The jet density remains approximately constant except near the jet front.

The Weibel instability remains excited by the continuously injected jet particles and the electromagnetic fields are maintained at a high level, about four times that seen in a previous, much shorter grid simulation system (with $\left.L_{\mathrm{x}}=640 \Delta\right)$. At earlier simulation times a large electromagnetic structure is generated and accelerates the ambient plasma. As shown in Fig. 1b, at this later simulation time the strong magnetic field extends up to $x / \Delta=2,000$. These strong fields become small in the shocked ambient region beyond $x / \Delta=$ 2000 (Nishikawa et al., 2006; Ramirez-Ruiz, Nishikawa, \& Hededal, 2007). 
The acceleration of ambient electrons becomes visible when jet electrons pass about $x / \Delta=500$. The maximum density of accelerated ambient electrons is attained at $t=1750 \omega_{\mathrm{pe}}^{-1}$. The maximum density gradually reaches a plateau as seen in Fig. 1a. The maximum electromagnetic field energy is located at $x / \Delta=1,700$ as shown in Fig. $1 \mathrm{~b}$.

\subsection{A Numerical Method for Calculating Emission}

Let a particle be at position $\mathbf{r}_{0}(t)$ at time $t$ (Nishikawa et al., 2008; Hededal, 2005; Hededal \& Nordlund, 2005). At the same time, we observe the electric field from the particle from position $\mathbf{r}$. However, because of the finite velocity of light, we observe the particle at an earlier position $\mathbf{r}_{\mathbf{0}}\left(\mathrm{t}^{\prime}\right)$ where it was at the retarded time $t^{\prime}=t-\delta t^{\prime}=t-\mathbf{R}\left(\mathrm{t}^{\prime}\right) / \mathrm{c}$. Here $\mathbf{R}\left(\mathrm{t}^{\prime}\right)=\left|\mathbf{r}-\mathbf{r}_{\mathbf{0}}\left(\mathrm{t}^{\prime}\right)\right|$ is the distance from the charge (at the retarded time $t^{\prime}$ ) to the observer.

After some calculation and simplifying assumptions the total energy $W$ radiated per unit solid angle per unit frequency from a charged particle moving with instantaneous velocity $\boldsymbol{\beta}$ under acceleration $\dot{\boldsymbol{\beta}}$ can be expressed as (Rybicki \& Lightman, 1979; Jackson, 1999)

$$
\frac{d^{2} W}{d \Omega d \omega}=\frac{\mu_{0} c q^{2}}{16 \pi^{3}}\left|\int_{-\infty}^{\infty} \frac{\mathbf{n} \times[(\mathbf{n}-\boldsymbol{\beta}) \times \dot{\boldsymbol{\beta}}]}{(1-\boldsymbol{\beta} \cdot \mathbf{n})^{2}} e^{i \omega\left(t^{\prime}-\mathbf{n} \cdot \mathbf{r}_{\mathbf{0}}\left(t^{\prime}\right) / \mathrm{c}\right)} d t^{\prime}\right|^{2}
$$

Here, $\mathbf{n} \equiv \mathbf{R}\left(\mathrm{t}^{\prime}\right) /\left|\mathbf{R}\left(\mathrm{t}^{\prime}\right)\right|$ is a unit vector that points from the particle's retarded position towards the observer.

The observer's viewing angle is set by the choice of $\mathbf{n}\left(n_{\mathrm{x}}^{2}+n_{\mathrm{y}}^{2}+n_{\mathrm{z}}^{2}=1\right)$. The choice of unit vector $\mathbf{n}$ along the direction of propagation of the jet (hereafter taken to be the $x$-axis) corresponds to head-on emission. For any other choice of $\mathbf{n}\left(\right.$ e.g., $\left.\theta_{\gamma}=1 / \gamma=\left(1-\beta^{2}\right)^{1 / 2}=\left(1-(\boldsymbol{v} / c)^{2}\right)^{1 / 2}\right)$, off-axis emission is seen by the observer. It is noted that in this article that radiative losses are not included (e.g., Jaroschek \& Hoshino, 2009; Reville, \& Kirk, 2010).

In order to calculate radiation from relativistic jets propagating along the $x$-direction (Nishikawa et al., 2008) we consider a test case which includes a parallel magnetic field $\left(B_{\mathrm{x}}\right)$, and jet velocity of $v_{\mathrm{j} 1,2}=0.99 \mathrm{c}$. Two electrons are injected with different perpendicular velocities $\left(v_{\perp 1}=0.1 c, v_{\perp 2}=0.12 c\right)$. A maximum Lorentz factor of $\gamma_{\max }=\left\{\left(1-\left(v_{\mathrm{j} 2}^{2}+v_{\perp 2}^{2}\right) / c^{2}\right\}^{-1 / 2}=13.48\right.$ is calculated for the larger perpendicular velocity.

Figure 2 shows electron trajectories in the $x-y$ plane (red: $v_{\perp 2}=0.12 c$, blue: $\left.v_{\perp 1}=0.1 c\right)$ (a: left panel), the radiation (retarded) electric field (b: middle panel), and spectra (c: right panel) for $B_{\mathrm{x}}=3.70$. The two electrons are propagating from left to right with gyration in the $y-z$ plane (not shown). The gyroradius is about $0.44 \Delta$ for the electron with the larger perpendicular 
velocity. The power spectrum is shown at seven viewing angles with respect to the $x$-direction of $0^{\circ}$ (red), $10^{\circ}$ (orange), $20^{\circ}$ (yellow), $30^{\circ}$ (moss green), $45^{\circ}$ (green), $70^{\circ}$ (light blue), and $90^{\circ}$ (blue). The higher frequencies become stronger at the $10^{\circ}$ viewing angle. The critical angle for off-axis radiation $\theta_{\gamma}=180^{\circ} /\left(\pi \gamma_{\max }\right)$ for this case is $4.25^{\circ}$. As shown in this panel, the spectrum at a larger viewing angle $\left(>20^{\circ}\right)$ has smaller amplitude. Only two different
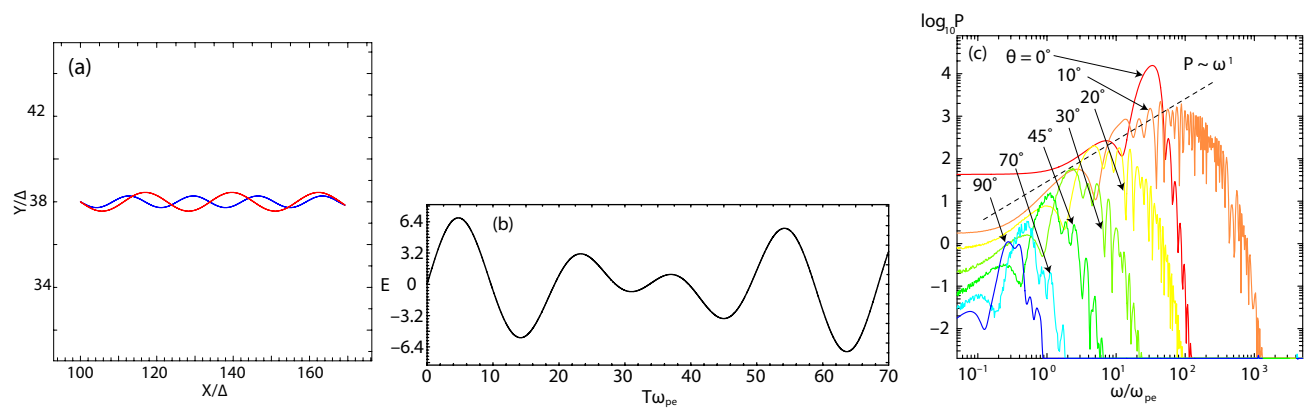

Fig. 2. A test case with a strong magnetic field $\left(B_{\mathrm{x}}=3.7\right)$ and two electrons with different perpendicular velocities $\left(v_{\perp 1}=0.1 c, v_{\perp 2}=0.12 c\right)$. (a) The paths of the helically moving electrons along the $x$-direction in the homogenous magnetic field are shown in the $x-y$-plane. (b) The two electrons radiate a time dependent electric field. The retarded electric field from the moving electrons seen by an observer situated in the rest frame at great distance along the n-vector. (c) The observed power spectrum at different viewing angles from the two electrons. Frequency is in units of $\omega_{\mathrm{pe}}^{-1}$.

electrons are used to calculate the radiation, therefore two cyclotron frequencies and their higher harmonics are visible in Fig. 2c, and the spectra are not smooth. Since the jet plasma has a large velocity $x$-component in the simulation frame, the radiation from the particles (electrons and positrons) is strongly beamed along the $x$-axis (as in jitter radiation) (Epstein, 1973; Medvedev, 2000, 2006).

Equations 6.30a and 6.30b in Rybicki \& Lightman (1979) are an approximation suggesting that radiation at the viewing angle $\alpha=0^{\circ}$ (eq. 6.33) disappears (see Fig. 6.5 in the textbook of Rybicki \& Lightman (1979)). However, an exact expression shows that radiation at viewing angle $0^{\circ}$ does not vanish (Bekefi, 1966; Landau \& Lifshitz, 1980) as a result of the relativistic distortion of the lobes of a Doppler-boosted dipole antenna pattern. This aspect is shown in Fig. 2c, and note that the amplitude at higher frequency at the viewing angle $10^{\circ}$ is stronger than at viewing angle $0^{\circ}$.

/Hoshino

\subsection{The Standard Synchrotron Radiation Model}

A synchrotron shock model is widely adopted to describe the radiation thought to be responsible for observed broad-band GRB afterglows (Zhang \& Meszaros, 
2004; Piran, 2005a,b; Zhang, 2007; Nakar, 2007). Associated with this model are three major assumptions that are adopted in almost all current GRB afterglow models. Firstly, electrons are assumed to be "Fermi" accelerated at the relativistic shocks and to have a power-law distribution with a powerlaw index $p$ upon acceleration, i.e., $N\left(E_{\mathrm{e}}\right) d E_{\mathrm{e}} \propto E^{-p} d E_{\mathrm{e}}$. This is consistent with recent PIC simulations of shock formation and particle acceleration (Spitkovskv, 2008b) and also some Monte Carlo models (Achterberg et al., 2001; Ellison \& Double, 2002; Lemoine \& Pelletier, 2003), but see (Niemiec, \& Ostrowski, 2006; Niemiec, Ostrowski, \& Pohl, 2006). Secondly, a fraction $\epsilon_{\mathrm{e}}$ (generally taken to be $\leq 1$ ) of the electrons associated with ISM baryons are accelerated, and the total electron energy is a fraction $\epsilon_{\mathrm{e}}$ of the total internal energy in the shocked region. Thirdly, the strength of the magnetic fields in the shocked region is unknown, but its energy density $\left(B^{2} / 8 \pi\right)$ is assumed to be a fraction $\epsilon_{B}$ of the internal energy. These assumed "micro-physics" parameters, $p, \epsilon_{\mathrm{e}}$ and $\epsilon_{\mathrm{B}}$, whose values are obtained from spectral fits (Panaitescu, \& Kumar, 2001; Yost et al., 2003) reflect the lack of a detailed description of the microphysics (Waxman, 2006).

The typical observed emission frequency from an electron with (comoving) energy $\gamma_{\mathrm{e}} m_{\mathrm{e}} c^{2}$ in a frame with a bulk Lorentz factor $\Gamma$ is $\nu=\Gamma \gamma_{\mathrm{e}}^{2}\left(e B / 2 \pi m_{\mathrm{e}} c\right)$. Three critical frequencies are defined by three characteristic electron energies. These are $\nu_{\mathrm{m}}$ (the injection frequency), $\nu_{\mathrm{c}}$ (the cooling frequency), and $\nu_{\mathrm{M}}$ (the maximum synchrotron frequency). In our simulations of GRB afterglows, there is one additional relevant frequency, $\nu_{\mathrm{a}}$, due to synchrotron self-absorption at lower frequencies (Meszaros, Rees, \& Wijer, 1998; Sari, Piran, \& Narayan, 1998; Zhang, 2007; Nakar, 2007).

The general agreement between blast wave dynamics and direct measurements of the fireball size argue for the validity of this model (Zhang, 2007; Nakar, 2007). The shock is most likely collisionless, i.e., mediated by plasma instabilities (Waxman, 2006). The electromagnetic instabilities mediating the afterglow shock are expected to generate magnetic fields. Afterglow radiation was therefore predicted to result from synchrotron emission of shock accelerated electrons (Meszaros \& Rees, 1997). The observed spectrum of afterglow radiation is indeed remarkably consistent with synchrotron emission of electrons accelerated to a power-law distribution, providing support for the standard afterglow model based on synchrotron emission of shock accelerated electrons (Piran, 1999, 2000, 2005a; Zhang \& Meszaros, 2004; Meszaros, 2002, 2006; Zhang, 2007; Nakar, 2007).

In order to determine the luminosity and spectrum of synchrotron radiation, the strength of the magnetic field $\left(\epsilon_{\mathrm{B}}\right)$ and the index of the electron energy distribution $(p)$ must be determined. Due to the lack of a first principles theory of collisionless shocks, a purely phenomenological approach to the model of afterglow radiation was ascribed without investigating in detail the 
processes responsible for particle acceleration and magnetic field generation (Waxman, 2006). It is important to clarify here that the constraints implied on these parameters by the observations are independent of any assumptions regarding the nature of the afterglow shock and the processes responsible for particle acceleration or magnetic field generation. Any model should satisfy these observational constraints.

The properties of synchrotron (or "jitter") emission from relativistic shocks will be determined by the magnetic field strength and structure and the electron energy distribution behind the shock. The characteristics of jitter radiation may be important to understanding the complex time evolution and/or spectral structure in gamma-ray bursts (Preece et al., 1998). For example, jitter radiation has been proposed as a means to explain GRB spectra below the peak frequency that are harder than the "line of death" spectral index associated with synchrotron emission (Epstein, 1973; Medvedev, 2000, 2006), i.e., the observed spectral power scales as $F_{\nu} \propto \nu^{2 / 3}$, whereas synchrotron spectra are $F_{\nu} \propto \nu^{1 / 3}$ or softer (Medvedev, 2006). Thus, it is essential to calculate radiation production by tracing electrons (positrons) in self-consistently generated small-scale electromagnetic fields.

\subsection{Calculating Synchrotron/Jitter Emission from Electron Trajectories in Self-consistently Generated Magnetic Fields}

In order to obtain the spectrum of synchrotron (jitter) emission, we consider an ensemble of electrons selected in the region where the Weibel instability has fully grown and electrons are accelerated in the generated magnetic fields. In order to validate our numerical method we performed simulations using a small system with $\left(L_{\mathrm{x}}, L_{\mathrm{y}}, L_{\mathrm{z}}\right)=(645 \Delta, 131 \Delta, 131 \Delta)(\Delta=1$ : grid size $)$ and a total of $\sim 0.5$ billion particles (12 particles/cell/species for the ambient plasma) in the active grid zones (Nishikawa et al., 2006). First we performed simulations without calculating radiation up to $t=450 \omega_{\mathrm{pe}}^{-1}$ when the jet front is located at about $x / \Delta=480$. We randomly selected 12,150 electrons in both the jet and in the ambient medium. Recently, a similar calculations have been carried out for the radiation from electrons accelerated in laser-wakefield acceleration (Martins et al., 2009), in counter-streaming jets (Frederiksen et al., 2010), and from a single shock in the contact discontinuity frame (Sironi \& Spitkovsky, 2009b).

Figure 3 shows (a) the current filaments generated by the Weibel instability and (b) the phase space of $x / \Delta-\gamma V_{\mathrm{x}}$ for jet electrons (red) and ambient electrons (blue) at $t=450 \omega_{\mathrm{pe}}^{-1}$. Figure 4 shows (a) the $x$-component of current density generated by the Weibel instability and (b) the phase space of jet electrons and ambient electrons at a slightly later time $t=525 \omega_{\mathrm{pe}}^{-1}$.

We calculated the emission from the jet and ambient 12,150 electrons during the sampling time $t_{\mathrm{s}}=t_{2}-t_{1}=75 \omega_{\mathrm{pe}}^{-1}$ with Nyquist frequency $\omega_{\mathrm{N}}=1 / 2 \Delta t=$ 

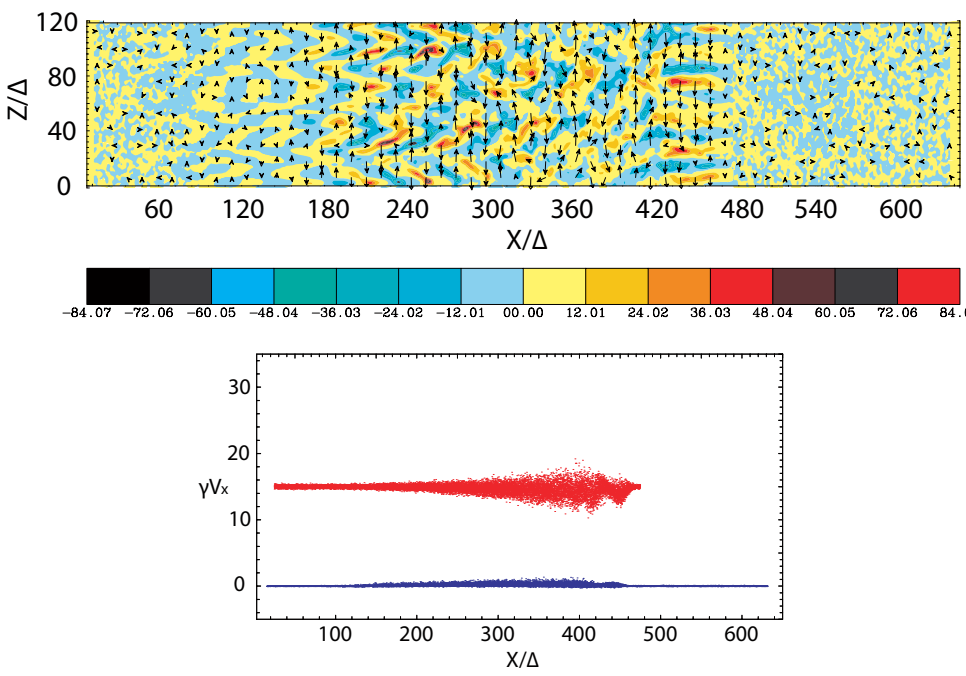

Fig. 3. Two-dimensional images in the $x-z$ plane at $y / \Delta=65$ for $t=450 \omega_{\mathrm{pe}}^{-1}$. The colors indicate the $\mathrm{x}$-component of current density generated by the Weibel instability, with the $\mathrm{x}$ - and $\mathrm{z}$-components of magnetic field represented by arrows (a). Phase space distributions as a function of $x / \Delta-\gamma v_{\mathrm{x}}$ plotted for the jet (red) and ambient (blue) electrons at the same time.

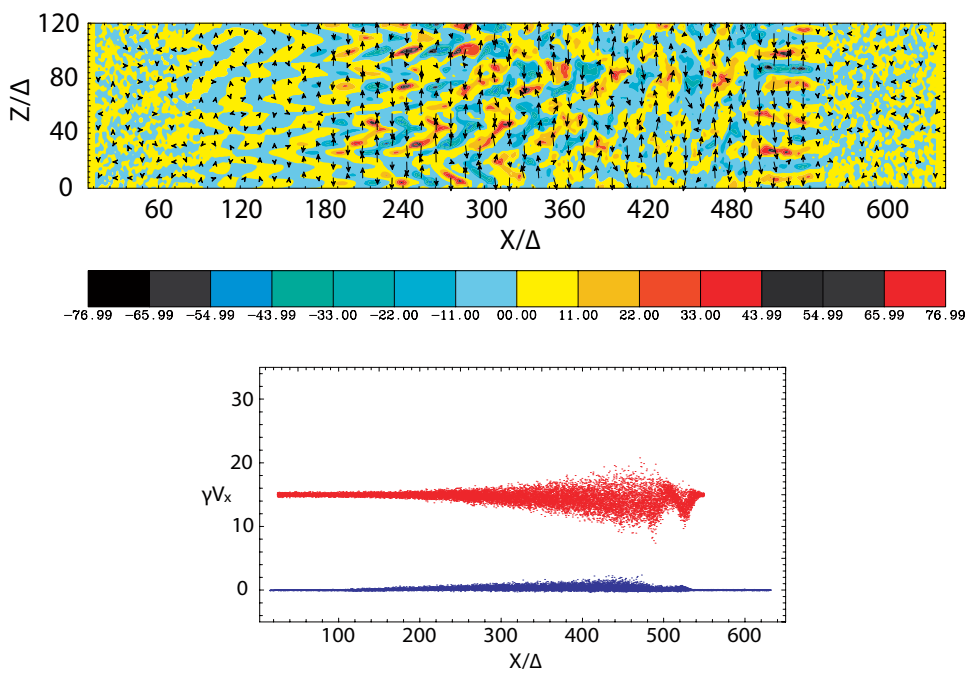

Fig. 4. Two-dimensional images in the $x-z$ plane at $y / \Delta=65$ for $t=525 \omega_{\mathrm{pe}}^{-1}$. The colors indicate the $\mathrm{x}$-component of current density generated by the Weibel instability, with the $\mathrm{x}$ - and $\mathrm{z}$-components of magnetic field represented by arrows (a). Phase space distributions as a function of $x / \Delta-\gamma v_{\mathrm{x}}$ plotted for the jet (red) and ambient (blue) electrons at the same time.

$200 \omega_{\mathrm{pe}}$ where $\Delta t=0.005 \omega_{\mathrm{pe}}^{-1}$ is the simulation time step and the frequency resolution $\Delta \omega=1 / t_{\mathrm{s}}=0.0133 \omega_{\mathrm{pe}}$. The resulting spectra shown in Figure 5 show emission from jet electrons and ambient electrons separately, and are calculated for head-on $\left(0^{\circ}\right)$ and $5^{\circ}$ viewing directions. The radiation from jet electrons shows Bremsstrahlung-like spectra as the red $\left(0^{\circ}\right)$ and orange 
$\left(5^{\circ}\right)$ lines (Hededal, 2005). The jet electron spectra are different from the spectra shown in Fig. 2c because the jet electrons are not much accelerated, the magnetic fields generated by the Weibel instability are rather weak and the electron trajectories are only slightly bent.

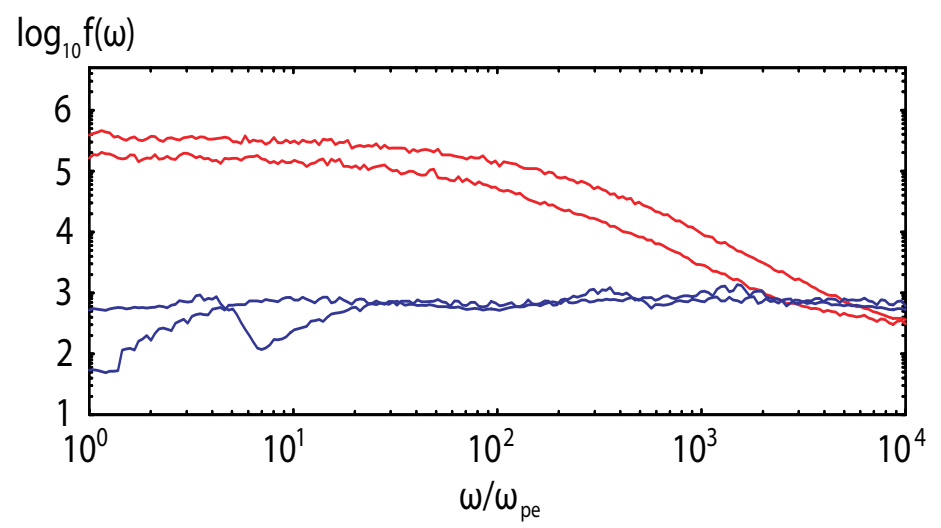

Fig. 5. Spectra obtained from jet and ambient electrons for the two viewing angles. Spectra for jet electrons are shown by red $\left(0^{\circ}\right)$ and orange $\left(5^{\circ}\right)$ lines. Spectra for ambient electrons are shown by blue $\left(0^{\circ}\right)$ and light blue $\left(5^{\circ}\right)$ lines.

We can compare the spectra in Fig. 5 with spectra obtained for two electrons as shown in Fig. 6. Here we have a parallel magnetic field, $B_{\mathrm{x}}=0.37$, a jet velocity of $v_{\mathrm{j} 1,2}=0.99 c$, and two electrons with different perpendicular velocities $\left(v_{\perp 1}=0.01 c, v_{\perp 2}=0.012 c\right)$. A maximum Lorentz factor of $\gamma_{\max }=\left\{\left(1-\left(v_{\mathrm{j} 2}^{2}+v_{\perp 2}^{2}\right) / c^{2}\right\}^{-1 / 2}=7.114\right.$ accompanies the larger perpendicular velocity. The critical angle for off-axis radiation $\theta_{\gamma}=180^{\circ} /\left(\pi \gamma_{\max }\right)$ for this case is $8.05^{\circ}$. A comparison between Fig. 5 and Fig. 6c indicates similarities.
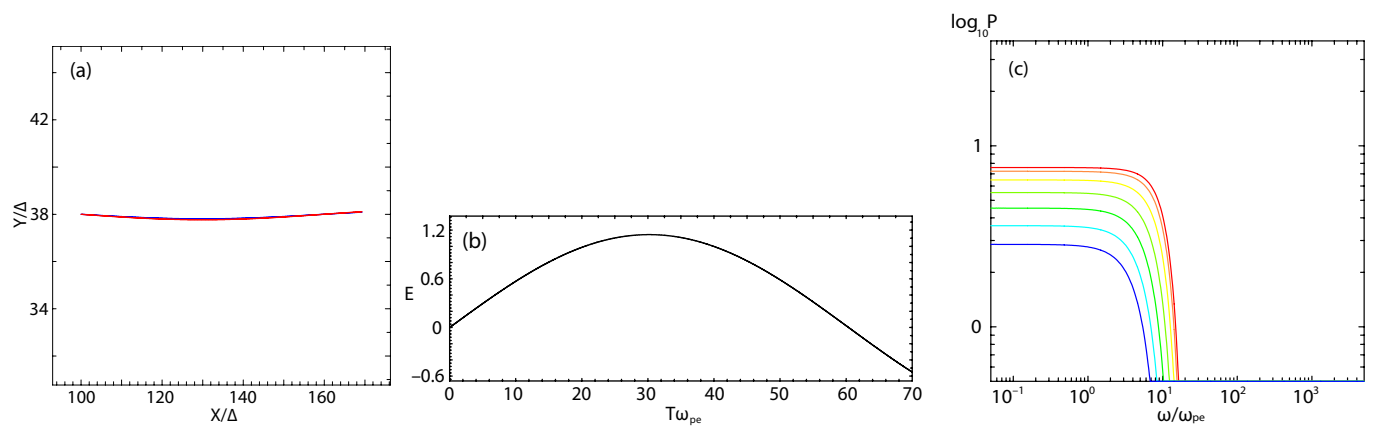

Fig. 6. The case with a weak magnetic field $\left(B_{\mathrm{x}}=0.37\right)$ and small perpendicular velocity $\left(v_{\perp 1}=0.01 c, v_{\perp 2}=0.012 c\right)$. The paths of two electrons moving helically along the $x$-direction in a homogenous magnetic field shown in the $x-y$-plane (a). The two electrons radiate a time dependent electric field. An observer situated at great distance along the n-vector sees the retarded electric field from the moving electrons (b). The observed power spectrum at different viewing angles from the two electrons (c). Frequency is in units of $\omega_{\text {pe }}$. It should be noted that the cyclotron frequency is around $20 \omega_{\mathrm{pe}}^{-1}$. 
The lower frequencies have flat spectra and the higher frequencies decrease monotonically. The slope in Fig. 5 is less steep than in Fig. 6c. This is due to the fact that the spread of Lorentz factors of the jet electrons is larger and the average Lorentz factor is larger as well. Although the magnetic field is not as strong in the simulation spectra, the spectra are extended to higher frequency. This is explained that as shown in Fig. 7.16 (left) in Hededal?s Ph. D. thesis (Hededal, 2005) the turbulent magnetic field shifts the frequency higher with shorter wave length (smaller $\mu$ ). We obtained results for other simulations with different parameters for the jet electrons and including an ambient magnetic field. In all cases the strength of the magnetic fields generated by the Weibel instability was small, and the spectra for these cases were very similar to Fig. 5. The low level for the magnetic field energy in these small test case simulations with $x / \Delta<600$ is to be expected. As indicated by Fig. 1b, the magnetic field energy in the region $x / \Delta<500$ is small $\left(\epsilon_{\mathrm{B}}<0.07\right)$, therefore, as expected, the spectra should look like that from electrons propagating in a turbulent magnetic field with some high frequency enhancement.

\section{Discussion}

Emission obtained with the method described above is self-consistent, and automatically accounts for magnetic field structures on the small scales responsible for jitter emission. By performing such calculations for simulations with different initial parameters, we can investigate and compare the different regimes of jitter- and synchrotron-type emission (Epstein, 1973; Medvedev, 2000, 2006). The feasibility of this approach has already been demonstrated (Hededal, 2005; Hededal \& Nordlund, 2005), and its implementation is straightforward. Thus, we should be able to address the low frequency GRB spectral index violation of the synchrotron spectrum line of death (Medvedev, 2006).

Recently, synthetic radiation has been obtained from electrons accelerated in laser-wakefield acceleration (Martins et al., 2009), in counter-streaming jets (Frederiksen et al., 2010), and from a single shock in the contact discontinuity frame (Sironi \& Spitkovsky, 2009b). Our simulation setup is different from these simulations in that we do not have a fixed contact discontinuity (CD) (reflected at the wall) (e.g. Sironi \& Spitkovskv, 2009b), or counter-streaming jets (e.g. Martins et al., 2009; Frederiksen et al., 2010). Instead we have been performing (e.g. Nishikawa et al., 2009) simulations like that shown in Fig. 1 , where we inject relativistic jets into an ambient plasma. A double shock structure (bow and jet shocks separated by a contact discontinuity region) is formed and electrons can be accelerated due to the Weibel instability in both shocks. Since we calculate the radiation from the electrons in the observer frame, and calculated spectra can be compared directly with observations. As shown in Fig. 1, the strongest electron acceleration and strongest magnetic fields are generated in the jet (trailing) shock. Therefore, in this simulation this region would produce the emission that is observed. 
Medvedev and Spitkovsky recently showed that electrons may cool efficiently at or near the shock jump and are capable of emitting a large fraction of the shock energy (Medvedev \& Spitkovsky, 2009). Such shocks are wellresolved in existing PIC simulations; therefore, the microscopic structure can be studied in detail. Since most of the emission in such shocks would originate from the vicinity of the shock, the spectral power of the emitted radiation can be directly obtained from finite-length simulations and compared with observational data. We will calculate more spectra from RPIC simulations and compare in detail with Fermi data in future work.

\section{Achknowledgments}

This work is supported by NSF-AST-0506719, AST-0506666, AST-0908040, AST-0908010, NASA-NNG05GK73G, NNX07AJ88G, NNX08AG83G, NNX 08AL39G, and NNX09AD16G. JN was supported by MNiSW research projects 1 P03D 00329 and N N203 393034, and The Foundation for Polish Science through the HOMING program, which is supported through the EEA Financial Mechanism.Simulations were performed at the Columbia facility at the NASA Advanced Supercomputing (NAS). and IBM p690 (Copper) at the National Center for Supercomputing Applications (NCSA) which is supported by the NSF. Part of this work was done while K.-I. N. was visiting the Niels Bohr Institute. Support from the Danish Natural Science Research Council is gratefully acknowledged. This report was finalized during the program "Particle Acceleration in Astrophysical Plasmas" at the Kavli Institute for Theoretical Physics which is supported by the National Science Foundation under Grant No. PHY05-51164.

\section{References}

Achterberg, A., Gallant, Y. A. Kirk, J. G., \& Guthmann, A. X., Particle acceleration by ultrarelativistic shocks: theory and simulations, MNRAS, 328, 393 - 408, 2001.

Bekefi, G., Radiative Processes in Plasmas, John Wiley \& Sons, New York, 1966.

Chang, P., Spitkovsky, A., \& Arons, J., Long-term evolution of magnetic turbulence in relativistic collisionless shocks: Electron-positron plasmas, ApJ, 674, 378 - 387, 2008.

Ellison, D. C., \& Double, G. P., Nonlinear particle acceleration in relativistic shocks, Astroparticle Phys., 18, 213 - 228, 2002.

Epstein, R. I., Synchrotron sources. I. Extension of theory for small pitch angles, ApJ, 183, 593 - 610, 1973.

Frederiksen, J. T., Haugbølle, T., Medvedev, M. V., \& Nordlund, Å., Radiation spectral synthesis of relativistic filamentation, ApJ, 722, L114 - L119, 2010.

Hededal, C.B., Ph.D. thesis, Gamma-Ray Bursts, Collisionless Shocks and Synthetic Spectra, 2005. (arXiv:astro-ph/0506559)

Hededal, C.B., \& Nordlund, A., Gamma-ray burst synthetic spec- 
tra from collisionless shock PIC simulaitons, ApJL, submitted, 2005. (arXiv:astro-ph/0511662)

Hededal, C. B., \& Nishikawa, K.-I., The influence of an Ambient Magnetic field on Relativistic Collisionless Plasma Shocks, ApJ, 623, L89 - L92, 2005. Jackson, J. D., Classical Electrodynamics, Interscience, 1999.

Jaroschek C. H. \& Hoshino, M., Radiation-Dominated Relativistic Current Sheets, PRL, 103, 075002 1-4, 2009.

Landau, L. D., \& Lifshitz, E. M., The Classical Theory of Fields, Elsevier Science \& Technology Books, 1980.

Lemoine, M., \& Pelletier, G., Particle Transport in Tangled Magnetic Fields and Fermi Acceleration at Relativistic Shocks, ApJ, 589, L73 - L76, 2003.

Martins, J.L., Martins, S.F., Fonseca, R.A. Silva, L.O., Radiation postprocessing in PIC codes Proc. of SPIE, 7359, 73590V-1 - 8, 2009.

Medvedev, M. V., \& Loeb, A., Generation of magnetic fields in the relativistic shock of gamma-ray burst sources, ApJ, 526, 697 - 706, 1999.

Medvedev, M. V., Theory of "Jitter" Radiation from Small-Scale Random Magnetic Fields and Prompt Emission from Gamma-Ray Burst Shocks, ApJ, 540, 704 - 714, 2000.

Medvedev, M. V., The theory of spectral evolution of the GRB prompt emission, ApJ, 637, 869 - 872, 2006.

Medvedev, M.V., \& Spitkovsky, A., Radiative cooling in relativistic collisionless shocks. Can simulations and experiments probe relevant GRB physics?, ApJ, 700, 956 - 964, 2009.

Meszaros, P., Theories of Gamma-Ray Bursts, ARAA, 40, 137 - 169, 2002.

Meszaros, P., Gamma-Ray Bursts, Rept. Prog. Phys., 69, 2259 - 2321, 2006.

Meszaros, P., \& Rees, M. J., Poynting Jets from Black Holes and Cosmological Gamma-Ray Bursts, ApJ, 482, L29 - L32, 1997.

Meszaros, P., Rees, M. J., Wijers, R. A. M. J., Viewing Angle and Environment Effects in Gamma-Ray Bursts: Sources of Afterglow Diversity, ApJ, 499, 301 - 308, 1998.

Nakar, E., Short-hard gamma-ray bursts, Phys. Reports, 442, 166 - 236, 2007.

Niemiec, J., \& Ostrowski, M., Cosmic Ray Acceleration at Ultrarelativistic Shock Waves: Effects of a "Realistic" Magnetic Field Structure, ApJ, 641, 984 - 992, 2006.

Niemiec, J., Ostrowski, M., Pohl, M., Cosmic-Ray Acceleration at Ultrarelativistic Shock Waves: Effects of Downstream Short-Wave Turbulence, ApJ, 650, 1020 - 1027, 2006.

Nishikawa, K.-I., Hardee, P., Richardson, G., Preece, R., Sol, H., \& Fishman, G. J., Particle Acceleration in Relativistic Jets due to Weibel Instability, ApJ, 595, 555 - 563, 2003.

Nishikawa, K.-I., Hardee, P., Richardson, G., Preece, R., Sol, H., \& Fishman, G. J., Particle Acceleration and Magnetic Field Generation in ElectronPositron Relativistic Shocks, ApJ, 623, 927 - 937, 2005.

Nishikawa, K.-I., Hardee, P., Hededal, C. B., \& Fishman, G. J., Acceleration Mechanics in Relativistic Shocks by the Weibel Instability, ApJ, 642, 1267 
- 1274, 2006.

Nishikawa, K. -I., Niemiec, J., Sol, H., Medvedev, M., Zhang, B., Nordlund, Å., Frederiksen, J. T., Hardee, P., Mizuno, Y., Hartmann, D., \& Fishman, G. J., New Relativistic Particle-In-Cell Simulation Studies of Prompt and Early Afterglows from GRBs, in Proceedings of The 4 th Heidelberg International Symposium on High Energy Gamma-Ray Astronomy, eds, F. A. Aharonian, W. Hofmann, F. Rieger 1085, 589 - 593, 2009. (arXiv:0809.5067)

Nishikawa, K.-I., Niemiec, J., Hardee, P. E., Medvedev, M. Sol, H., Mizuno, Y., Zhang, B., Pohl, M., Oka, M., \& Hartmann, D. H., Weibel instability and associated strong fields in a fully $3 \mathrm{D}$ simulation of a relativistic shock, ApJ, 689, L10 - L13, 2009.

Panaitescu, A., \& Kumar, P., Fundamental Physical Parameters of Collimated Gamma-Ray Burst Afterglows, ApJ, 560, L49 - L52, 2001.

Piran, T., Gamma-ray bursts and the fireball model, Phys. Rep., 314, 575 667, 1999.

Piran, T., Gamma-ray bursts - a puzzle being resolved, Phys. Rep., 333, 529 - 553, 2000.

Piran, T., The physics of gamma-ray bursts, Rev. Mod. Phys., 76, 1143 - 1210, 2005a.

Piran, T., Magnetic Fields in Gamma-Ray Bursts: A Short Overview, in the proceedings of Magnetic Fields in the Universe, Angra dos Reis, Brazil, Nov. 29-Dec 3, 2004, Ed. E. de Gouveia del Pino, AIPC, 784, 164 - 174, 2005b.

Preece, R. D., Briggs, M. S., Mallozzi, R. S., Pendleton, G. N., Paciesas, W. S., \& Band, D. L., The Synchrotron Shock Model Confronts a "Line of Death" in the BATSE Gamma-Ray Burst Data, ApJ, 506, L23 - L26, 1998.

Ramirez-Ruiz, E., Nishikawa, K.-I., \& Hededal, C. B., $\mathrm{e}^{ \pm}$Loading and the origin of the upstream magnetic field in GRB shocks, ApJ, 671, 1877 1885, 2007.

Reville, B. \& Kirk, J. G., Computation of Synthetic Spectra from Simulations of Relativistic Shocks, ApJ, 724, 1283 - 1295, 2010.

Rybicki, G. B., \& Lightman, A. P., Radiative Processes in Astrophysics, John Wiley \& Sons, New York, 1979.

Sari, R., Piran, T., Narayan, R., Spectra and Light Curves of Gamma-Ray Burst Afterglows, ApJ, 497, L17 - L20, 1998.

Sironi, L., \& Spitkovsky, A., Particle acceleration in relativistic magnetized collisionless paslas: Dependence of shock acceleration on magnetic obliquity, ApJ, 698, 1523 - 1549, 2009a.

Sironi, L. \& Spitkovsky, A., Synthetic Spectra from PIC simulations of relativistic collisionless shocks, ApJ, 707, L92L96, 2009b.

Spitkovsky, A., On the structure of relativistic collisionless shocks in electronion plasmas, ApJ, 673, L39 - L42, 2008a.

Spitkovsky, A., Particle acceleration in relativistic collisionless shocks: Fermi process at last, ApJ, 682, L5 - L8, 2008b.

Waxman, E., Gamma-ray bursts and collisionless shocks, Plasma Phys. Control. Fusion, 48, B137 - B151, 2006. (arXiv:astro-ph/0607353) 
Yost, S. A., Harrison, F. A., Sari, R., Frail, D. A., A Study of the Afterglows of Four Gamma-Ray Bursts: Constraining the Explosion and Fireball Model, ApJ, 597, 459 - 473, 2003.

Zhang, B., \& Meszaros, P., Gamma-Ray Bursts: progress, problems \& prospects, Int. J. Mod. Phys., A19, 2385 - 2472, 2004.

Zhang, B., Gamma-Ray Bursts in the Swift Era, Chin. J. Astron. Astrophys., $7,1-50,2007$. 